\title{
A World-Wide Net of Solar Radio Spectrometers e-CALLISTO
}

\section{Conference Paper}

Author(s):

Benz, A. O.; Monstein, C.; Meyer, H.; Manoharan, P. K.; Ramesh, R.; Altyntsev, A.; Lara, A.; Paez, J.; Cho, K.-S.

Publication date:

2009-04

Permanent link:

https://doi.org/10.3929/ethz-b-000157338

Rights / license:

In Copyright - Non-Commercial Use Permitted

Originally published in:

Earth, Moon, and Planets 104(1-4), https://doi.org/10.1007/s11038-008-9267-6 


\title{
A World-Wide Net of Solar Radio Spectrometers: e-CALLISTO
}

\author{
A. O. Benz $\cdot$ C. Monstein $\cdot$ H. Meyer $\cdot$ P. K. Manoharan $\cdot$ \\ R. Ramesh $\cdot$ A. Altyntsev $\cdot$ A. Lara $\cdot$ J. Paez $\cdot$ K.-S. Cho
}

Received: 13 November 2007/ Accepted: 10 April 2008/Published online: 13 January 2009

(C) Springer Science+Business Media B.V. 2009

\begin{abstract}
Radio spectrometers of the CALLISTO type to observe solar flares have been distributed to nine locations around the globe. The instruments observe automatically, their data is collected every day via internet and stored in a central data base. A public webinterface exists through which data can be browsed and retrieved. The nine instruments form a network called e-CALLISTO. It is still growing in the number of stations, as redundancy is desirable for full $24 \mathrm{~h}$ coverage of the solar radio emission in the meter and low decimeter band. The e-CALLISTO system has already proven to be a valuable new tool for monitoring solar activity and for space weather research.
\end{abstract}

Keywords Solar radio emission - Radio spectrometer - International science cooperation - Global network - Space weather - International Heliospheric Year

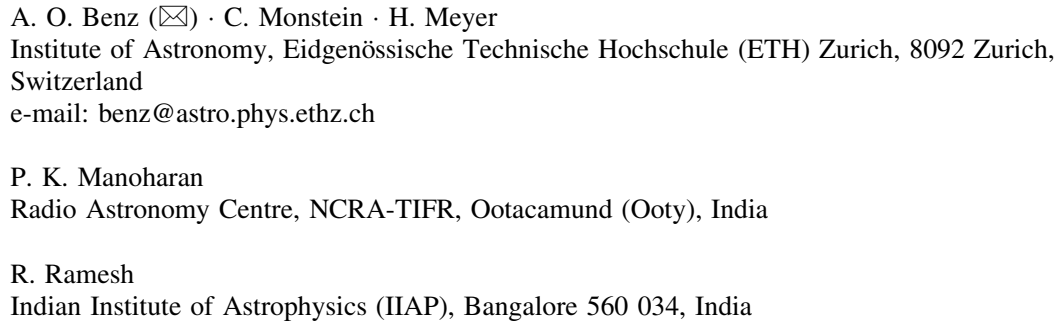




\section{Introduction}

The investigation of the Sun has made great progress in the past decade due to observations from satellites and space probes in solar particles, X-rays, extreme UV and by white-light coronal emission. However, the dynamics of the solar corona is still not understood, and new phenomena are unveiled every year. It is no surprise that the plasma phenomena emitting radio waves are little or not at all known. Thus the solar radio emission as observed from groundbased telescopes at meter and decimeter wavelengths poses still many challenges and potential treasures after many decades of study. Understanding radio emission can only progress by combination with observations at other wavelengths from space. There are only few groundbased solar radio instruments that survey a large part of the spectrum. Presently, the full coverage of the solar radio spectrum is limited to daylight in Europe, whereas space probes observe fulltime. For large flares and coronal mass ejections (CMEs), lasting several hours, this limits the number of joint events. In addition, important events are often only partially observed, and the preflare record is usually not complete. As a result, there exist only few observations of large events in their entire duration.

Radio waves at meter and decimeter waves can be used as a diagnostics of the solar processes (review by McLean and Labrum 1985; theory review by Benz 2002). They provide the first signature of flare shocks (Type II events) and electron beams escaping from the Sun on open magnetic field lines (Type III bursts). In addition, they record unexplained emissions of active regions (Type I noise storms) and long lasting emission after large flares (Type IV events). Most recent interest focuses on the predictive potential of radio emission regarding CMEs heading toward Earth (review by Gopalswamy 2006). They greatly disturb our local space weather and are potential hazards for space missions.

Here we report on an endeavour, termed e-CALLISTO, initialized in the frame of the International Heliospheric Year (IHY) that was announced by the United Nation Organization (UNO) for 2007. The e-CALLISTO network aims at $24 \mathrm{~h}$ coverage of the radio emission of the Sun. The goal is to spread identical spectrographs in the meter wave and low decimeter radio band around the globe and connect them through the internet. The e-CALLISTO project is based on a spectrometer unit, provided by ETH Zurich, as well as an antenna and a PC interface to the internet, provided by the local partners. The Compact Astronomical Low-cost Low-frequency Instrument for Spectroscopy and Transportable Observatory (CALLISTO) has been described in detail by Benz et al. (2005). Here we report on the international set-up of a distributed net of CALLISTO spectrometers, data acquisition by internet and first results of the system.

\section{Instruments}

The CALLISTO spectrometer is composed of a handful of standard electronic components available from the consumer market and few others from eBay assembled on a single PCB (printed circuit board, Fig. 1). This PCB fits into a standard aluminum box and has connectors for the antenna, computer, power supply, focal plane unit and-as an option-to an external $1 \mathrm{MHz}$ clock source. The main specifications are listed in Table 1. A complete set of drawings, parts list, procedures, PCB-layout and also the complete software is freely available on the internet (for the address, see link at the end of paper). Up to now, almost all CALLISTO spectrometers have been assembled by young apprentices (mechanics) of the ETH physics department. Each Callisto was completely tested using an automated test 
Table 1 Main specifications of the instrument

\begin{tabular}{ll}
\hline Parameter & Specification \\
\hline Frequency range & $45.0-870.0 \mathrm{MHz}$ (in three sub-bands) \\
Frequency resolution & $62.5 \mathrm{KHz}$ \\
Radiometric bandwidth & $300 \mathrm{KHz}$ at $-3 \mathrm{~dB}$ \\
Dynamic range & $\sim 50 \mathrm{~dB}$ at -70 to $-30 \mathrm{dBm}$ maximum rf level \\
Sensitivity & $25 \pm 1 \mathrm{mV} / \mathrm{dB}$ \\
Noise figure & $<10 \mathrm{~dB}$ (measured at the rf input connector) \\
Maximum sampling rate & Internal clock $800 \mathrm{~S} / \mathrm{s}$, external clock $1,000 \mathrm{~S} / \mathrm{s}$ \\
Number of channels & Selectable $1-500$, nominal 200 frequencies per sweep \\
Power supply & DC $12 \pm 2 \mathrm{~V} / 225 \mathrm{~mA}$ \\
Weight & $\sim 800 \mathrm{~g}$ \\
Dimensions & $110 \mathrm{~mm} \times 80 \mathrm{~mm} \times 205 \mathrm{~mm}$ \\
Material cost & $<200 \mathrm{US} \$$ \\
Input data & Three files (configuration, frequency, scheduler) \\
Output data & Two files (one FITS-file per 15 min and one log file per day) \\
\hline
\end{tabular}

setup, controlled by a PC connected to a programmable radio signal generator via IEEE488 interface bus. The most important test results of every manufactured CALLISTO are also available on the internet.

The spectrometers have been shipped or carried by an ETH engineer to the host institutes. The list of sites is given in Table 2 and the worldwide distribution displayed in Fig. 2. An important first step was a site evaluation, revealing sometimes unexpected interference by nearby radio transmitters. A spectral overview was made using a special function of CALLISTO for every host site. It allows measuring the whole frequency range from 45 to $870 \mathrm{MHz}$ in steps of $62.5 \mathrm{KHz}$ leading to potentially 13,120 channels. This high resolution spectrum is then used to create a dedicated frequency program avoiding channels with terrestrial interference. Such a frequency program observes only those

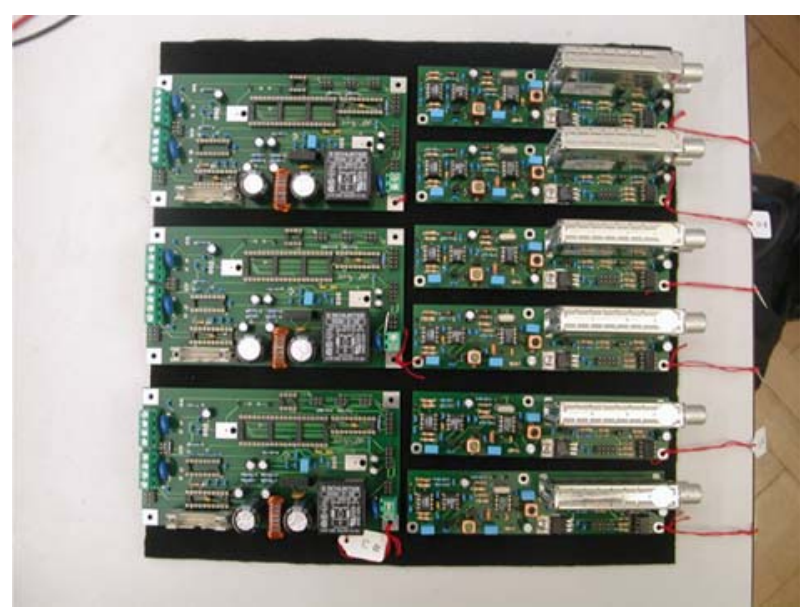

Fig. 1 Three CALLISTO printed circuit boards assembled by mechanics apprentices at ETH Zurich 
Table 2 Key data of worldwide distributed and hitherto installed and configured host sites sorted by time of delivery

\begin{tabular}{|c|c|c|c|c|c|c|}
\hline Country & Place & Institute & Longitude & Latitude & Antenna & Polarization \\
\hline Switzerland & Bleien & ETH/RSG & $8.11 \mathrm{E}$ & $47.34 \mathrm{~N}$ & Log-per & $1 \times$ linear $45^{\circ}$ \\
\hline Switzerland & Zurich & ETH/STS & $8.55 \mathrm{E}$ & $47.37 \mathrm{~N}$ & Dish $5 \mathrm{~m}$ & $1 \times \mathrm{RHCP}$ \\
\hline India & Ootacamund & TIFR & $76.7 \mathrm{E}$ & $11.4 \mathrm{~N}$ & Log-per & $2 \times$ linear $\mathrm{z}$ \\
\hline India & Gauribidanur & IIAP & $77.5 \mathrm{E}$ & $13.6 \mathrm{~N}$ & Log-per & $1 \times$ linear $\mathrm{z}$ \\
\hline Russian Fed. & Badary & ISTP & $102.2 \mathrm{E}$ & $51.7 \mathrm{~N}$ & Log-per & $1 \times$ linear $\mathrm{x}$ \\
\hline Costa Rica & San Jose & CINESPA & $84 \mathrm{~W}$ & $10 \mathrm{~N}$ & Dish $7 \mathrm{~m}$ & $1 \times$ linear \\
\hline Mexico & D. F. & UNAM & $99 \mathrm{~W}$ & $19 \mathrm{~N}$ & Log-per & $1 \times$ linear $\mathrm{H}$ \\
\hline Switzerland & Freienbach & Private & $8.76 \mathrm{E}$ & $47.20 \mathrm{~N}$ & Log-per & $1 \times$ linear $\mathrm{H}$ \\
\hline South Korea & Daejeon & KASI & $127.4 \mathrm{E}$ & $36.4 \mathrm{~N}$ & Log-per & $1 \times$ linear $\mathrm{H}$ \\
\hline
\end{tabular}

Polarization $\mathrm{z}$ means pointing to zenith with linear polarized antenna while the dipoles are pointing in east/ west-and/or north/south-direction. Polarization $\times$ means that it changes with time due to polar mount tracking system of the antenna. At noon it is horizontally polarized (ISTP Russia). H means horizontal polarization while RHCP denotes to right hand circular polarization and LHCP to left hand polarization

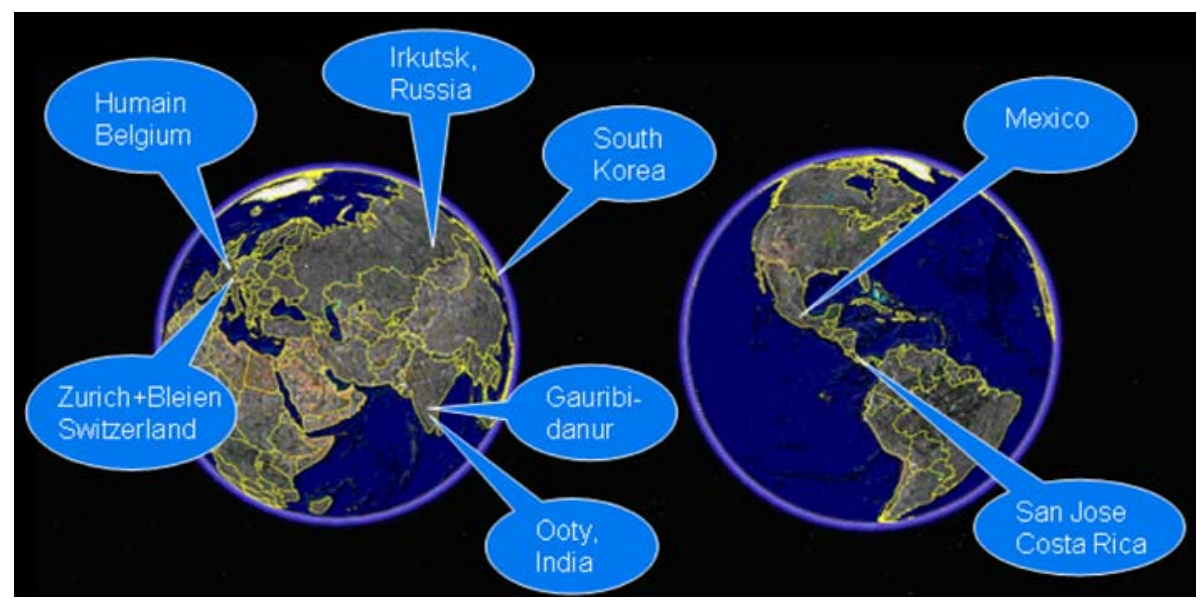

Fig. 2 Geographical distribution of CALLISTO host sites in October 2007. Humain in Belgium is in planning phase, while all other stations are delivering data to our database

frequencies with low radio frequency interference (rfi) and if needed it jumps over spectral ranges like the FM band between 80 and $110 \mathrm{MHz}$. Several spectral overviews have been made during the last years, the results are available online (see link below).

In most cases, the detected interference was home made by computers or switches. A major advantage of the CALLISTO instrument is the programmable maximum gain of the antenna input power in the range of $-70 \mathrm{dBm}$ up to $-30 \mathrm{dBm}$ to cope with the dissimilar level radio frequency interference at different locations.

About half of the present host sites have built there antennas from standard aluminum profiles in local workshops (Fig. 3). The other half procured broad-band radio amateur antennas from local stores. Such antennas are cheap but the lifetime in humid environment is limited to 1 or 2 years. A few host sites have access to professional commercial antennas that have guaranteed all-weather specification and long lasting life time. 

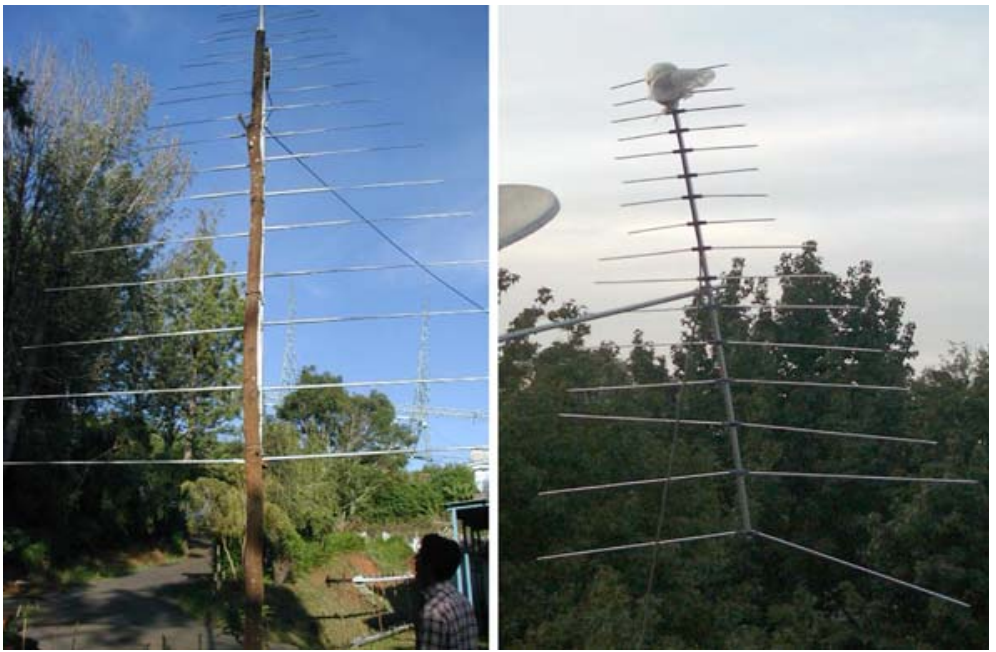

Fig. 3 Logarithmic periodic antennas of e-CALLISTO. Left: at Ootacamund (Ooty) in Tamil Nadu, India; Right: at UNAM, Mexico D.F.

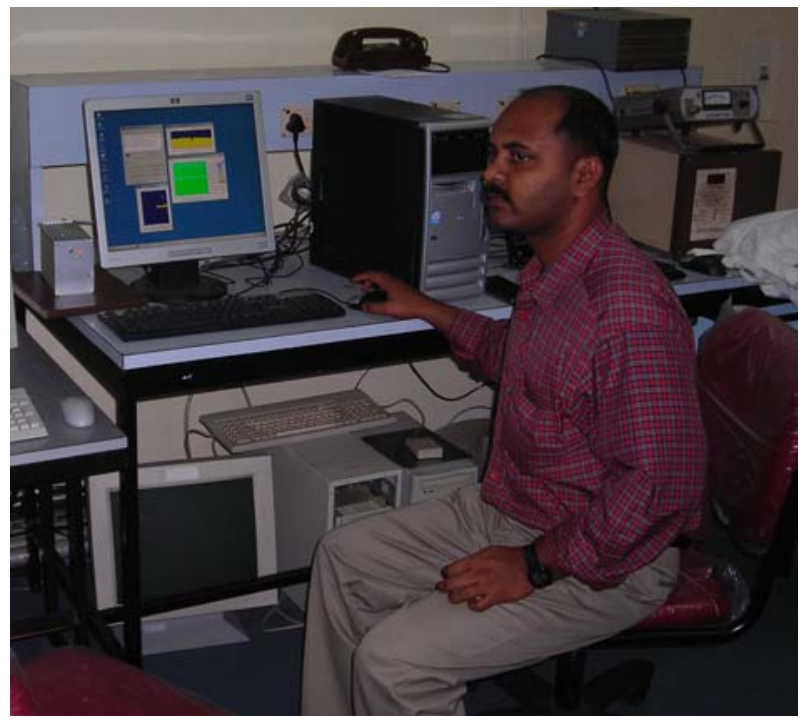

Fig. 4 C. Kathiravan of IIA at the CALLISTO console in the Gauribidanur radio observatory near Bangalore in India. The CALLISTO radio spectrometer is positioned to the left of the LCD screen

In general, there is no need for a permanent operator. Once the system is powered and configured it runs automatically, controlled by an internal scheduler of the PC. This scheduler allows automatic starting and stopping of observations as well as controlling of an optional focal plane unit with up to 64 different configurations. It is realized by a 6-bit digital output connected to a standard SUB-D25 connector. This option is needed for automatic calibration or antenna switching. An operator is only needed in case of missing 
Fig. 5 Hee Seon Rho, a young student of solar physics starts to assemble a CALLISTO spectrometer, brought to Korea in parts

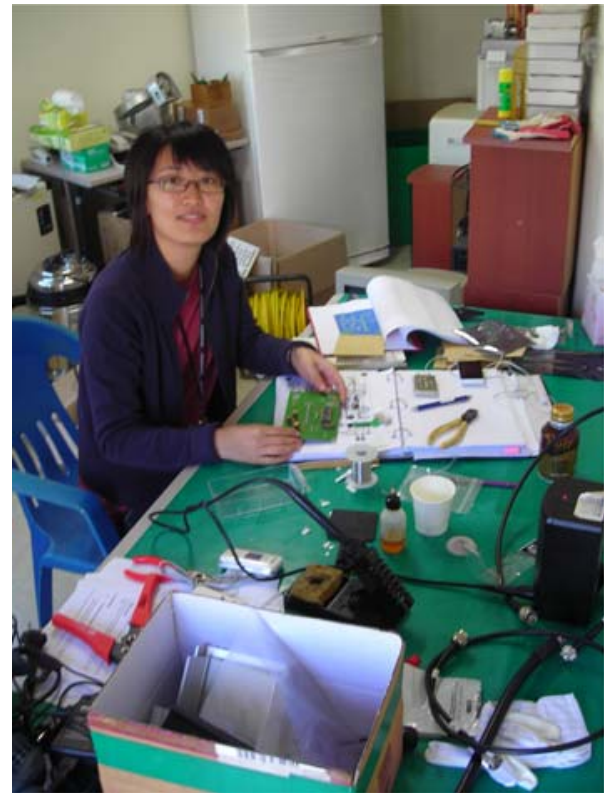

internet connection to transfer data manually to an external data server. Operators at Gauribidanur/India have to deal with this once per week by writing data to a compact disc (Fig. 4). Then the CD is transferred to the Indian Institute of Astrophysics, Bangalore, to copy the data onto the server. They are moved to an FTP server from where the data is introduced to our database at ETH Zurich.

At the Korea Astronomy and Space Science Institute (KASI) the installation of eCALLISTO was combined with an educational exercise. The ETH engineer, who traveled to South Korea to configure the main CALLISTO spectrometer, thought a student how to assemble a spectrometer from parts and to test the instrument. The student (Fig. 5) had to solder all these tiny SMD components onto the printed circuit board and to mount cabling and connectors to a complete spectrometer. This spare spectrometer is foreseen to do a survey in Korea regarding rfi. The same software which is used for the observation of solar radio flares can also be used to monitor rfi. Rfi monitoring is of general interest in today's radio polluted environment. This monitoring data may also be used to send reports about interfering emissions to the local administration.

Flux calibration has been performed by relating solar emission to observations by a calibrated instrument at the same frequency (e.g. Benz et al. 2005). This is a considerable effort and particularly difficult at frequencies $<100 \mathrm{MHz}$.

\section{Data Acquisition}

All data and log files of each host site between local sunrise and sunset are first stored on a local data disk. After sunset, a PERL script running on our server connects every host in all the countries and collects some files. Only data files relating to times of flares as reported in the list of NOAA (see link below) are transferred to our server, all others are ignored. It means that only data with a certain probability to contain a solar radio event are collected. 
The finally transferred and stored data are then sorted into a structured archive on our server having free access for everybody.

All available data are presented on a web site using a Java process called "Distributed Radio spectrometer Control" (DiRaC, http://www.astro.phys.ethz.ch:8080/frontend/). Using DiRaC, any date with reported flares between November 2006 (start of the IHYrelated project) and today can be selected. One of seven host sites must be selected with one or two polarizations (if available). By pressing the button "Get images", a direct presentation of the spectrogram covering $15 \mathrm{~min}$ of observation appears. The spectrogram presents all channels versus time in graphical form. By pressing the left or right arrow, an earlier or later data file is accessed. A mouse click on one of the thumb nail picture(s) gets an enlarged spectrogram. Selecting the zoom click box, a zoomed version appears after some time to prepare and to download from our server. A spectrogram on the screen may be downloaded in different graphics formats such as png or jpeg, or even the original FITS data files. The formats png and jpeg allow choosing the geometry of the output file. Since $\mathrm{DiRaC}$ is object oriented, several host sites of the same date can be loaded onto the screen allowing direct comparison with each other. The most important parameters of the host site are given, including a link to their web site.

\section{First Results}

The e-CALLISTO system has had its first success already in December 2006, when the last large flares of the present cycle occurred. These X-class flares where observed by the then newly launched Hinode satellite and may remain the only large flares observed by this satellite in the near future. Thus they are currently studied by several groups around the world. None of the flares were observed during the entire length from the ground due to the short December daylight in the Northern hemisphere. However, e-CALLISTO, then operating in Switzerland, India and Siberia, covered more than $60 \%$ of the time.

Figure 6 shows the solar flare radio emission of May 20, 2007 observed by the e-CALLISTO spectrometer element in Gauribidanur (India). The data is displayed as a spectrogram, intense emission presented bright, no emission black. There are 200 channels displayed horizontally, time progressing to the right. The pseudo three dimensional representations of time, frequency, and intensity produce an image that can be interpreted more easily. The frequency is given in $\mathrm{MHz}$, increasing downwards. As the emission is proportional to the density (plasma emission), the vertical axis also represents altitude in the solar atmosphere, comprising about one to two solar radii above the photosphere. As density decreases with altitude, height increases upward in the picture. The radio emission is tilted towards the right, indicating that an exciter is moving up-wards. Assuming a density model of the corona, the speed can be estimated. It amounts to about a third of the speed of light. The emission is therefore interpreted as the signature of an electron beam escaping from the Sun. Such events are known as Type III radio bursts. Note the low level of interference in Fig. 6. Only few terrestrial emissions are seen as horizontal lines.

The spectrogram of Fig. 7 displays the radio emission of a flare on May 23, 2007. The emission drifts again to the right, indicating an exciter moving upwards. However, the speed is much slower in this case and of the order of $1,000 \mathrm{~km} / \mathrm{s}$. This is a typical velocity of a shock wave in the corona. Such radio emissions, called Type II radio bursts, are often precursors of coronal mass ejections, which disturb the whole heliosphere and are of great interest for near-Earth space weather. 


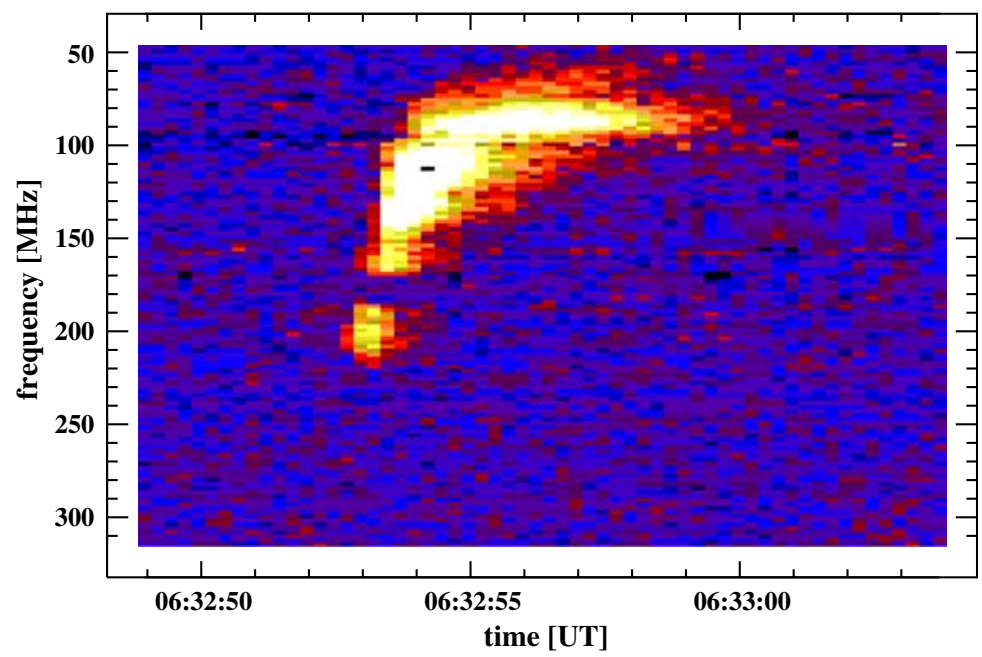

Fig. 6 Spectrogram of a radio event observed with e-CALLISTO spectrometer at the Gauribidanur/India

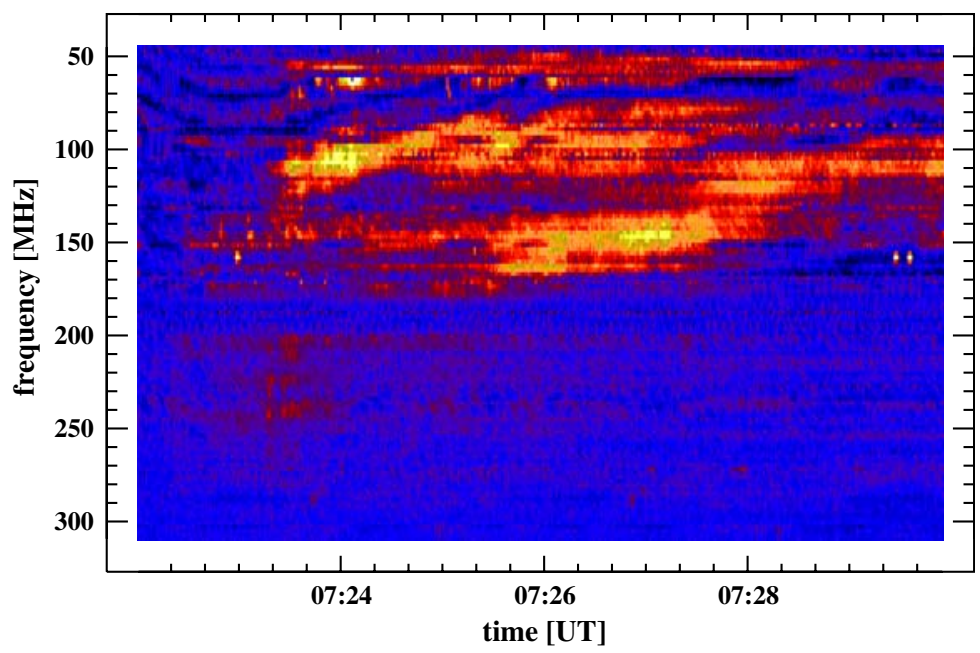

Fig. 7 Spectrogram of a radio event observed with e-CALLISTO spectrometer in Badary (Siberia), operated by the Institute of Solar-Terrestrial Physics (ISTP), Siberian Branch of Russian Academy of Sciences, Irkutsk, Russian Federation

\section{Conclusions}

The e-CALLISTO network is growing and produced first results. It has already shown its usefulness and delivered first results despite the currently low level of solar activity. The data is retrieved automatically from currently nine stations every day. Some stations have worked reliably for nearly one year. The interest of researchers to join the net is still great. More stations are planned, but limited by the availability of our engineers. Growth in the 
pacific area (see Fig. 2) is desirable from a scientific point of view. The e-CALLISTO network will be a reliable tool to observe and study solar activity during the upcoming ascending and maximum phases of cycle 24 . The learning effect and motivation of the people involved may be another justification, interesting to be evaluated in a few years.

\section{Links}

Documents: http://www.astro.phys.ethz.ch/instrument/callisto/ecallisto/applidocs.htm Data archive DiRaC: http://pandora.ethz.ch:8080/frontend/

Direct access to FITS files: http://www.astro.phys.ethz.ch/cgi-bin/showdir?dir= Observation_callisto\&file=dir.html

Spectral overviews: http://www.astro.phys.ethz.ch/instrument/callisto/RFSPEC2/spectrum2. htm

NOAA event list: http://www.sec.noaa.gov/ftpmenu/indices/events.html

Acknowledgements We would like to thank F. Aebersold and P. Behm (ETH), D. Nandagopal, R. Venkata-subramani, and A. J. Selvanayagam (Ooty), C. Kathiravan (IIA), S. V. Lesovoi and A. Maslov (SSRT), E. Mendoza and G. Paz (IGEF), M. Barantes (CINESPA), Su-Chan Bong, Hee-Seon Roh, Jakyoung Nah, and Han-Kyu Choi (KASI) for installation and technical support of e-CALLISTO. Omar Awyle and Christina Pöpper (both ETH) have written much of the software for data collection, presentation and archiving. The development, production and distribution of CALLISTO spectrometers it supported by the Swiss National Science Foundation (grant 2000220-113556), the Russian Foundation for Basic Research (grant 06-02-16295) and by the "Space Weather Center Project" of KASI and KASI Basic Research Fund.

\section{References}

A.O. Benz, Plasma Astrophysics, 2nd edn. (Springer, Dordrecht, 2002)

A.O. Benz, C. Monstein, H. Meyer, Solar Phys. 226, 143-151 (2005)

N. Gopalswamy, Radio Observations of Solar Eruptions, Solar Physics with the Nobeyama Radioheliograph, in Proceedings of Nobeyama Symposium, 2006, pp. 81-94

D.J. McLean, N.R. Labrum, Solar Radiophysics: Studies of Emission from the Sun at Metre Wavelengths (Cambridge University Press, Cambridge, 1985) 